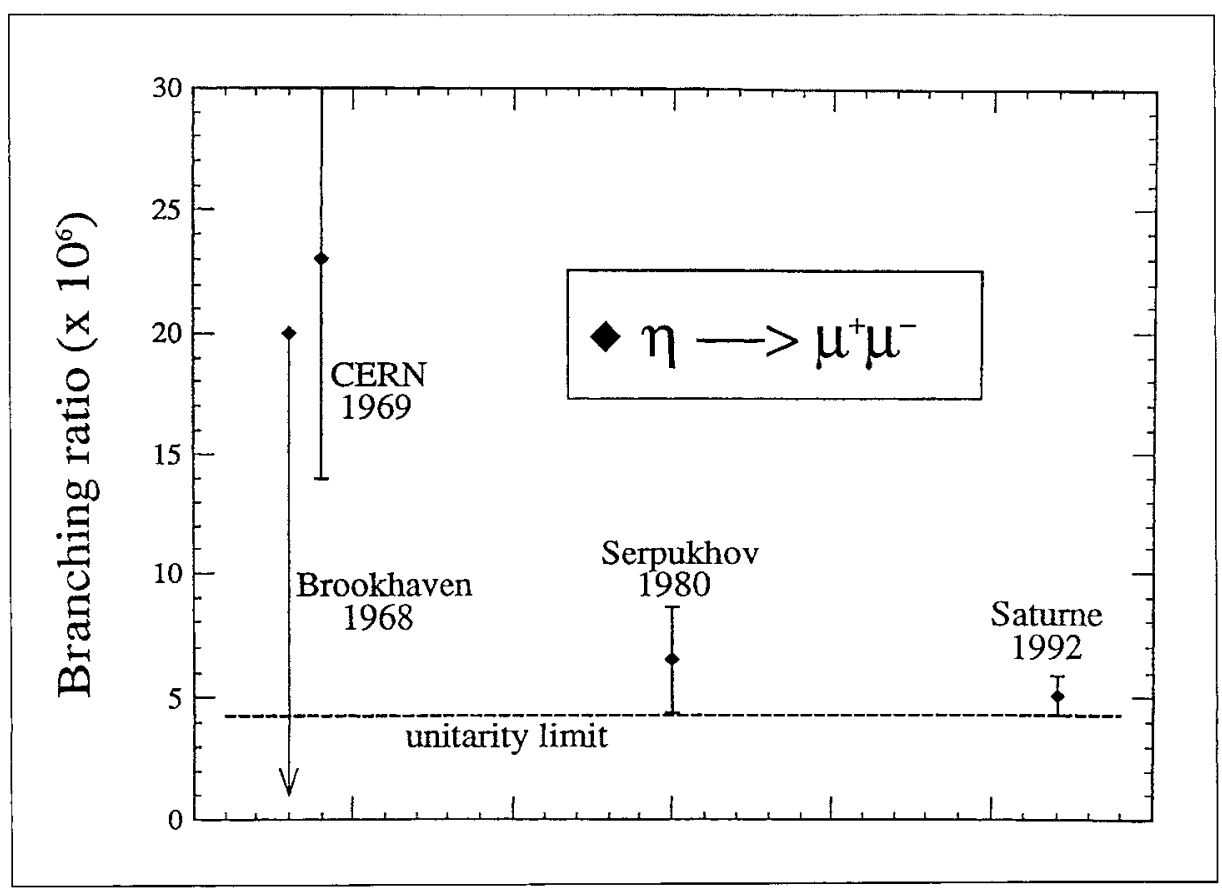

$10^{-9}$. Par exemple, les désintégrations de l'êta en un pion neutre et une paire de muons ou d'électrons constituent un test de l'invariance par conjugaison de charge au niveau d'un état intermédiaire à un photon; des désintégrations en paires électron-positon seraient une preuve (indirecte) de l'existence des leptoquarks et une désintégration en un muon et un électron démontrerait le mélange des familles leptoniques.

Le dispositif êta rend également possibles des mesures nouvelles et détaillées du facteur de forme de transition électromagnétique de l'êta par l'étude de sa désintégration en une paire de muons plus un photon et permet de sonder la théorie de perturbation par des mesures des désintégrations dans la voie à un pion neutre et deux photons.

\section{Intelligence artificielle}

L'intelligence artificielle et les domaines qui s'y rapportent fournissent un exemple éclatant du besoin croissant d'une technologie de pointe dans les expériences de physique les plus avancées. Le second séminaire international «Génie logiciel, intelligence artificielle et systèmes experts en physique des hautes énergies et physique nucléaire», qui s'est tenu du 13 au 18 janvier sur le site de FranceTélécom (Agelonde) à La Londe-lesMaures, Var, en a été l'illustration. C'était le deuxième de la série, le premier s'était tenu à Lyon en 1990 .

Quatre thèmes étroitement liés ont été abordés: le génie logiciel, les systèmes experts, les réseaux neuronaux et les techniques de manipulation symbolique.

Devant l'ampleur et la complexité des expériences qui se dessinent à l'horizon de la fin de ce siècle, il apparaît essentiel de mettre en application les techniques de l'intelligence artificielle (IA). Toutefois, des problèmes communs se présentent dans différents domaines (hautes énergies, nucléaire, plasma, spatial, télécommunications,...) et on recherche des solutions en mettant en place des collaborations laboratoire-industrie dans un cadre de coopération internationale.

Partant d'une identification des besoins réels de la recherche fondamentale mettant en oeuvre de gros équipements, cette approche mène d'une part à l'élaboration de techniques ou de produits nouveaux qu trouveront leur place dans le monde industriel et d'autre part à l'amélioration des méthodes de l'IA.

De nombreux spécialistes venus de domaines extérieurs à la physique des particules ont participé à ce séminaire: plusieurs d'entre eux ont joué un rôle d'avant-garde dans le domaine de l'IA. Des cours ont traité de la logique de la méthode des composants en génie logiciel (C. Vogel, directeur des méthodes avancées à la CISIIngéniérie, Paris), des langages à objets (EIFFEL) (J.M. Nerson, Société des outils du logiciel, Paris) et des applications utilisant plusieurs langages (M. Kunze, Université de la Ruhr).

Une introduction à l'IA et aux systèmes experts (D. Patridge, Université d'Exeter) a été suivie d'une description du langage PROLOG III (A. Colmerauer, Aix-Marseille, qui en est l'auteur), de présentations sur les possibilités des algorithmes génétiques (T.. Bach, Dortmund), sur l'emploi des réseaux neuronaux pour la reconnaissance de formes ( $F$.

Fogelman, Mimetics, Paris) et dans des applications en physique des hautes énergies ( $B$. Denby, FNAL).

Lors des sessions plénières, certains orateurs ont résumé les trois journées de séances parallèles, d'autres ont appuyé leurs exposés sur des programmes de physique en cours. Citons les orateurs suivants : $P$. Kunz (SLAC) décrivit l'informatique pour l'usine B au SLAC, Ch. Arnault (LAL) l'approche orientée objet dans Delphi, $R$. Jones (CERN) l'interface utilisateur graphique dans les systèmes d'acquisition, M.J. Ratcliffe (ECRC, Munich) les relations avec l'industrie et D.M. Sendall (CERN) qui concluait par un résumé de la session parallèle. C. Peterson (Lund) détaillait ensuite les résultats actuels des réseaux neuronaux en physique des hautes énergies, D. Seligson (INTEL) la réalisation électronique des $\mathrm{RN}, \mathrm{Ch}$. Kiesling (MPI Munich) et J.R. Hansen (Niels Bohr Institute) les applications en temps réel des RN sur les accélérateurs actuels et futurs (LHC/SSC). E. Wildner-Malendain (CERN) et K.H. Becks (Bergische Univ.) conclurent 


\section{USA advertisements in CERN COURIER}

Format A4

Monthly publication

All advertisements are published in both English and French editions. Second language versions accepted without extra charge.

Advertising Rates (US Dollars)

\begin{tabular}{|c|c|c|c|c|}
\hline Insertions & $\begin{array}{c}\text { Full } \\
\left(7 \frac{1}{2} \times 10^{1 / 2}\right)\end{array}$ & $\begin{array}{c}\text { Half } \\
\left(7^{1 / 4} \times 51 / 8\right)\end{array}$ & $\begin{array}{c}\text { Half } \\
\left(3 \frac{1}{2} \times 10^{1 / 2}\right)\end{array}$ & $\begin{array}{c}\text { Quarter } \\
\left(3 \frac{1}{2} \times 51 / 8\right)\end{array}$ \\
\hline 1 & 1625 & 960 & 960 & 561 \\
3 & 1558 & 905 & 905 & 520 \\
5 & 1500 & 855 & 855 & 485 \\
10 & 1420 & 790 & 790 & 450 \\
\hline
\end{tabular}

\section{Color Rates}

Each additional color

Haif page color

Covers 2 and 3

Cover 4 (back)

$\$ 1000$

$\$ 1500$

$\$ 1200$ (per color)

$\$ 1300$ (per color)

These prices include no entitlement to special placing.

Publication date

Closing date for

positive films and copy 1st of month preceding cover date

The cost of making films and of translation for advertisements are charged in addition (\$ 150 US). Advertisements cancelled after 1 st of month preceding cover

Screen (offset) date will be invoiced. 160

These rates are effective for the year 1992

Inquiries for USA and Japan:

Yvette M. Perez, Gordon and Breach Science Publishers, Frankford Arsenal, Bldg 110, 5301 Tacony Street, Box 330,

Philadelphia, PA 19137

Tel. : + 1 (215) 5377262

Fax: + 1 (215) 5370711

Inquiries for Europe please see page III.

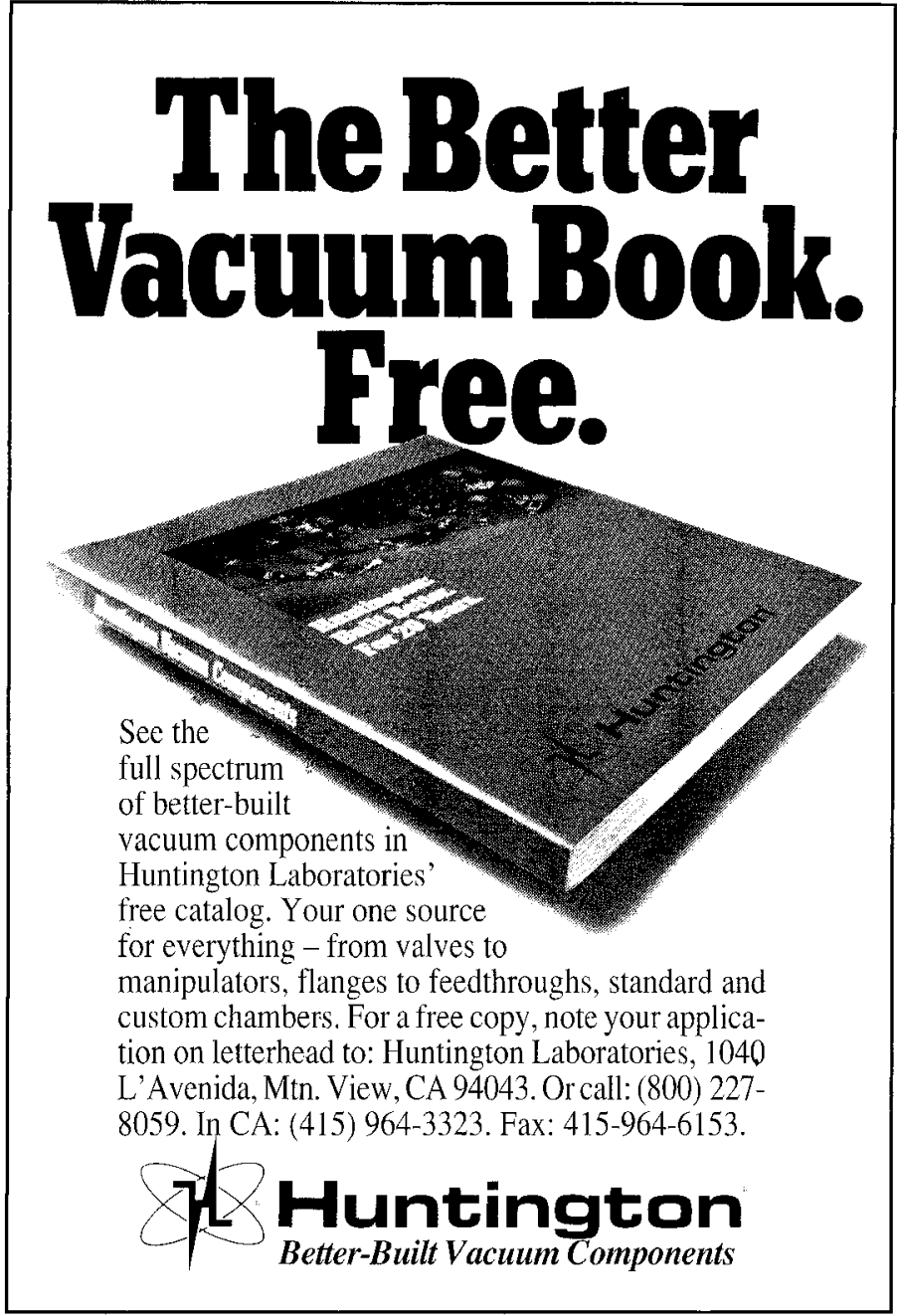

35 Circle advertisement number on reader service form

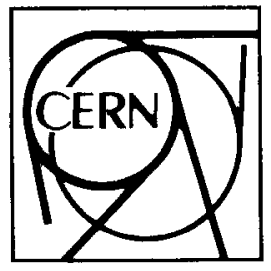

\section{How to visit CERN}

\section{Comment visiter le CERN}

Organized visits take place only on Saturdays, at 9.30 a.m., and/or 2.30 p.m. The visits last about three hours and are free. The minimum age limit is 16 years.

Les visites commentées ont lieu seulement le samedi, à $9 \mathrm{~h} .30$ et/ou à 14 h. 30. Elles durent environ trois heures et sont gratuites. La limite d'âge minimum imposée est de seize ans.

Please write or call: Ecrire ou téléphoner:
CERN

Visits Organization / Organisation des visites

1211 GENEVA 23 Switzerland

Tel. 022/767 4102

Telex 419000 CER 


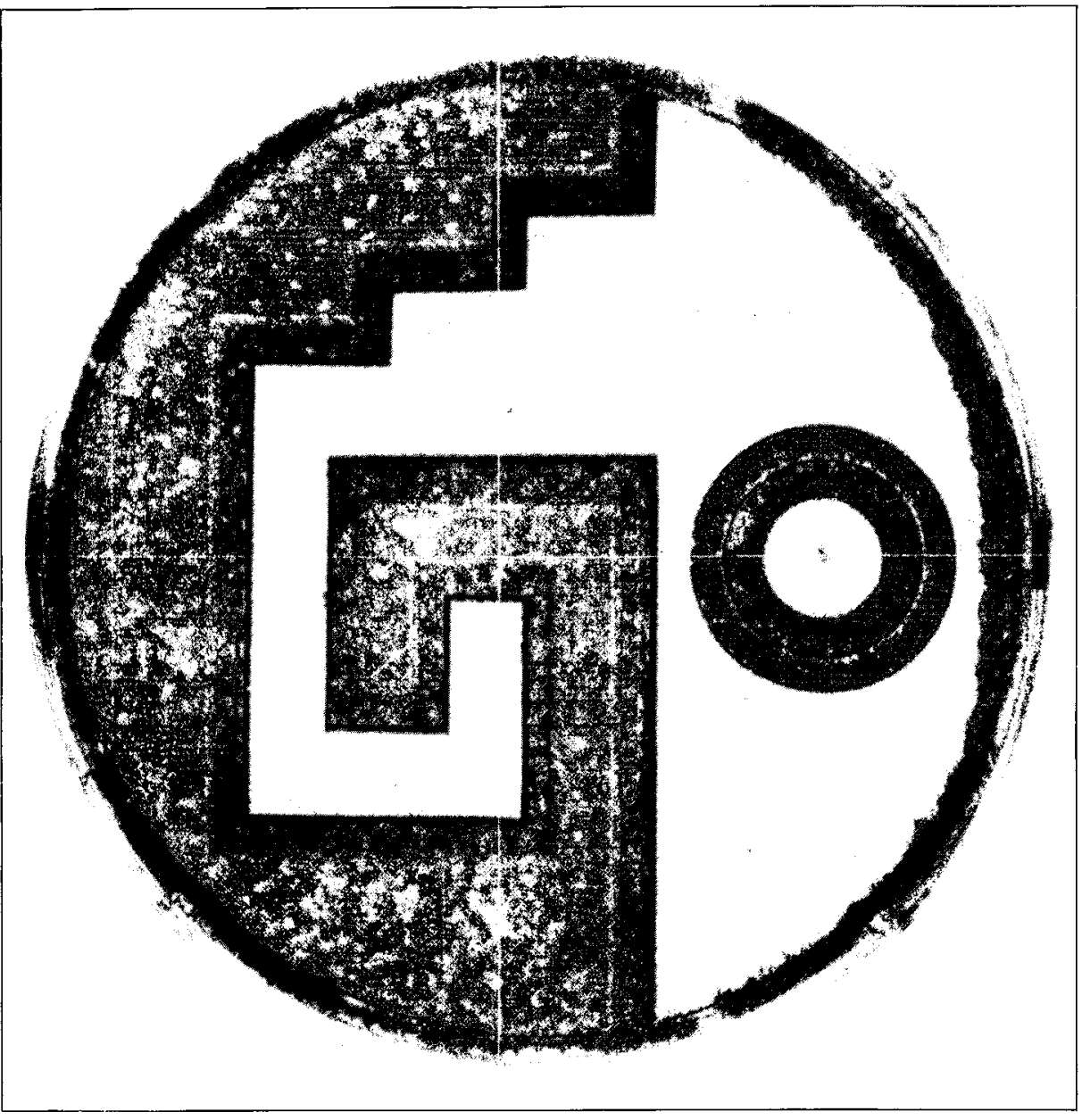

cette session.

Les techniques de manipulation symbolique permettent d'intégrer dans les méthodes de calcul l'évaluation algébrique des grandeurs mesurables et ainsi d'éviter les aspects fastidieux et pénibles des calculs numériques de précision, y compris sur les diagrammes de Feynman et les paramètres des accélérateurs. Les auteurs des principaux langages de manipulation symbolique présentèrent leurs plus récents résultats : G. Gonnet, ETHZ pour MAPLE, T. Hearn, Rand pour REDUCE, J. Vermaseren, Nikhef pour FORM. E. Remiddi, Bologne, démontra l'intérêt de SCHOONSCHIP et ASHMEDAI et K. Kaneko, Meiji Gakuin Univ résuma la session consacrée à ces techniques.

Plusieurs entreprises, en particulier France-Télécom et le CNET, ont participé avec des exposés et des présentations de leurs produits. Une liaison Numéris a permis d'effectuer des travaux d'application.

Un résultat essentiel a été la mise sur pied de groupes de travail (sous l'acronyme ASTEC) sur quatre thèmes principaux:

1 - établir ou maintenir un contact au niveau mondial entre les équipes travaillant sur les sujets abordés et permettre l'intégration de petits groupes dans les grands projets;

2 -structurer les relations avec l'industrie et d'autres domaines de recherche par la mise en place de projets communs;

3 -contribuer à la formation dans les techniques nouvelles;

4 -élaborer des propositions de standardisation du logiciel ou des protocoles d'interface entre les différentes applications.

Des serveurs de type LISTSERV sont installés sur CERNVM (ASTEC at CERNVM) pour permettre l'échange d'informations entre ces groupes et susciter l'intérêt pour ces techniques dans le reste de la communauté de physique nucléaire et des hautes énergies. Un Livre blanc synthétisant les résultats de ce travail sera publié.

Source : D. Perret-Gallix.

\section{KEK Deutons}

A la fin du mois de janvier, le synchrotron à protons (PS) de $12 \mathrm{GeV}$ au Laboratoire japonais KEK a réussi à accélérer des deutons à $11,2 \mathrm{GeV}$ (5,6 GeV/nucléon), l'énergie limite pour des deutons dans cet anneau. L'intensité du faisceau dans cet essai a dépassé $3 \times 10^{11}$ particules par impulsion.

Après les protons du PS et les électrons et positons du collisionneur TRISTAN, les deutons nouvellement arrivés ajoutent une autre option importante sur la carte des faisceaux disponibles au KEK.

Depuis son achèvement en 1976, le PS du KEK a fait l'objet d'améliorations constantes et il fournit des faisceaux de protons à diverses expériences. Quand au milieu des années 80 le projet TRISTAN a commencé à attirer de nombreux physiciens des hautes énergies, le programme expérimental du PS a effectué une transition et aujourd'hui il couvre un large domaine de recherches comprenant la physique nucléaire et atomique, les particules et les hadrons ainsi que la chimie nucléaire. C'est ce qui explique la demande récente de faisceaux de deutons de haute énergie.

La chaîne du PS au KEK comprend un pré-accélérateur Cockcroft-Walton de $750 \mathrm{keV}$, un linac Alvarez de $40 \mathrm{MeV}$ et un synchrotron injecteur de $500 \mathrm{MeV}$ alimentant l'anneau principal de 12 $\mathrm{GeV}$. Le faisceau qui reste disponible dans le synchrotron injecteur sert pour des études utilisant la diffusion des neutrons ou les muons ainsi que pour le traitement du cancer.

Pour produire des faisceaux de deutons, il a fallu apporter plusieurs 